\title{
INFLUENCE OF ACETYLCHOLINE ON THE PULMONARY ARTERIAL PRESSURE
}

\author{
BY \\ PETER HARRIS* \\ From the Cardiological Department, King's College Hospital \\ Received July 22, 1956
}

It is still a matter for debate whether active variation in the calibre of the vessels of the pulmonary circulation can be caused by physiological or pharmacological stimuli. In most observations, the agents used to test the pulmonary circulation are known to have an effect also on the systemic circulation and often on the cardiac output. Under these circumstances the pulmonary arterial pressure is liable to be influenced by factors other than local alterations in the calibre of the vessels of the lesser circulation.

In an attempt to circumvent these difficulties, it was decided to study the effects of acetylcholine on the pressures in the pulmonary circulation in a group of patients. Ellis and Weiss (1932) demonstrated the destruction of acetylcholine in the blood stream in man by the lack of cumulative action during its prolonged intravenous infusion. They and also Carmichael and Fraser (1933) observed flushing of the head and upper part of the body when acetylcholine was injected in sufficient dosage into a vein, while intra-arterial injection caused a regional dilatation of arteries and arterioles but no general effects. Since acetylcholine is destroyed so rapidly in the blood stream, it might be possible to inject it into the pulmonary artery in such a dose that it was inactivated before reaching the systemic circulation (Tiffenau and Beauvallet, 1944). In this way it was hoped that any vasomotor effect of the drug would be confined to the pulmonary circulation.

In animals, the action of acetylcholine is most evident in the systemic circulation, where it causes widespread arterial vasodilatation irrespective of whether these vessels are supplied by the sympathetic or parasympathetic systems (Hunt and Taveau, 1906; Dale, 1914; Hunt, 1917a and 1917b). Reports of the activity of the drug in the pulmonary circulation, however, have been conflicting (Hunt, 1917a; Daly, 1932 and 1933; von Euler, 1932; Franklin, 1932; Gaddum and Holtz, 1933; Johnson et al., 1937; Hamilton et al., 1939; Petrovskaia, 1939; Foggie, 1940; Friedberg et al., 1943; von Euler and Liljestrand, 1946; Dirken and Heemstra, 1948).

\section{METHODS}

Forty-seven patients were investigated. They had all been referred for diagnostic cardiac catheterization and had various disorders, which are given in Table I. Six of the children were anæsthetized with rectal pentothal: the remaining patients were conscious and received no premedication. All were in as near a basal state as possible. Three of the anæsthetized children had been given atropine.

A Cournand catheter was placed so that its tip lay in the main pulmonary artery. Between the hilt of the catheter and the head of the manometer was interposed a two-way tap with a short side-arm, through which a small volume of a solution of acetylcholine could be introduced into the lumen of the catheter. A recording of the pressure in the pulmonary artery was begun and, after an appropriate interval, the solution of acetylcholine was suddenly washed through the catheter into the pulmonary artery by means of a syringe filled with saline attached to the side-arm. With rapid manipulation of tap and syringe, the injection time was usually about two seconds. The recording of pressure was continued until the effects of the injection had disappeared or until it was apparent that there was no alteration in the pulmonary arterial pressure. In three cases the tip of the catheter lay in the right ventricle and then the right ventricular systolic pressure

* Work done during tenure of the Wiltshire Memorial Research Scholarship (1953-55) and submitted as part of a Ph.D. thesis at the University of London. 
TABLE I

Details of each Patient and the Effects of Acetylcholine

\begin{tabular}{|c|c|c|c|c|c|c|c|c|c|c|c|}
\hline \multirow[b]{2}{*}{$\begin{array}{l}\text { Case } \\
\text { No. }\end{array}$} & \multirow[b]{2}{*}{$\begin{array}{c}\text { Sex } \\
\text { and } \\
\text { age } \\
\text { (years) }\end{array}$} & \multirow[b]{2}{*}{ Diagnosis } & \multicolumn{4}{|c|}{ Before acetylcholine } & \multicolumn{5}{|c|}{ Effects of acetylcholine } \\
\hline & & & 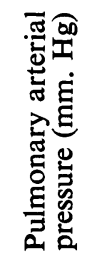 & 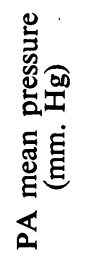 & 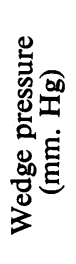 & 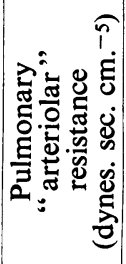 & 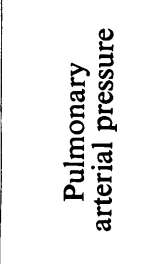 & 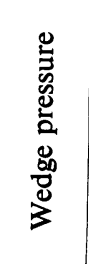 & 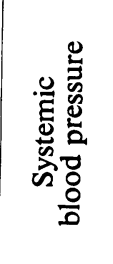 & $\begin{array}{l}\text { 量 } \\
\overrightarrow{0}\end{array}$ & 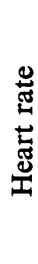 \\
\hline 1 & F 33 & MS & $57 / 28$ & 44 & 30 & 180 & 0 & +5 & - & 0 & 0 \\
\hline 2 & F 46 & MS & $115 / 50$ & $\begin{array}{l}44 \\
77\end{array}$ & 41 & 820 & $-20 / 12 \cdot 5$ & $\begin{array}{l}+3 \\
+2.5\end{array}$ & 二 & 0 & 0 \\
\hline 3 & F 43 & PDA & $140 / 10$ & 59 & 5 & 270 & $-42 \cdot 5 / 7 \cdot 5$ & - & - & 0 & 0 \\
\hline 4 & F 40 & MS. MI & $64 / 35$ & 50 & 33 & 290 & $-12 \cdot 5 / 10$ & +10 & 0 & 0 & 0 \\
\hline 5 & F 25 & MS. AI & $66 / 41$ & 53 & 37 & 150 & $-12 \cdot 5 / 7 \cdot 5$ & - & - & 0 & $\mathbf{0}$ \\
\hline 6 & F 33 & MS & $116 / 56$ & 82 & 46 & 770 & $-20 / 10$ & - & 0 & 0 & $\mathbf{0}$ \\
\hline 7 & F 8 & Normal & $34 / 18$ & 24 & 13 & - & 0 & - & - & 0 & 0 \\
\hline 8 & M41 & ASD & $170 / 68$ & 125 & - & - & 0 & - & - & 0 & 0 \\
\hline 9 & F 60 & PI. Emphysema & $68 / 25$ & 44 & - & - & $-20 / 15$ & - & 0 & 0 & $\mathbf{0}$ \\
\hline 10 & M 3 & VSD $\dagger$ & $85 / 52$ & 70 & 14 & . & $-22 \cdot 5 / 27.5$ & - & $-10 / 10$ & 0 & 0 \\
\hline 11 & M15 & Normal & $28 / 14$ & 19 & 16 & 100 & 0 & - & $-20 / 10$ & + & + \\
\hline 12 & M17 & VSD & $40 / 21$ & 29 & 18 & - & 0 & - & - & 0 & 0 \\
\hline 13 & M25 & MS & $28 / 11$ & 17 & 16 & 10 & 0 & $\mathbf{0}$ & - & 0 & 0 \\
\hline 14 & M39 & MS & $44 / 24$ & 33 & 30 & & 0 & 0 & - & 0 & 0 \\
\hline 15 & M56 & MI. A fibr. & $55 / 25$ & 46 & 31 & 350 & $-12 \cdot 5 / 0$ & - & - & 0 & $\mathbf{0}$ \\
\hline 16 & F 38 & MS & $88 / 33$ & 57 & 36 & 350 & $-15 / 10$ & - & 0 & + & + \\
\hline 17 & M42 & ASD & $155 / 58$ & 95 & - & & 0 & - & - & 0 & 0 \\
\hline 18 & M39 & Pulmo. Hypert. & $105 / 47$ & 67 & - & - & $-12 \cdot 5 / 5$ & - & - & 0 & 0 \\
\hline $19+$ & F 45 & MS. TI, A Fibr. & $124 / 57$ & 89 & - & - & 0 & - & - & 0 & 0 \\
\hline 20 & F 3 & PDA. ASD & $60 / 40$ & 51 & 9 & - & 0 & - & - & 0 & 0 \\
\hline 21 & M 4 & Cong. MS $\dagger$ & $50 / 22$ & 35 & 28 & - & 0 & - & - & 0 & 0 \\
\hline 22 & M50 & Emphysema & $39 / 22$ & 30 & 13 & 330 & 0 & 0 & $\mathbf{0}$ & 0 & 0 \\
\hline 23 & F 40 & Eisenmenger & $140 / 55$ & 93 & 4 & - & 0 & - & - & + & + \\
\hline 24 & F 30 & MS & $39 / 18$ & 28 & 24 & - & 0 & - & - & $\mathbf{0}$ & 0 \\
\hline 25 & F 40 & MS & $100 / 73$ & 84 & 32 & 480 & $-10 / 10$ & - & - & 0 & 0 \\
\hline 26 & F 9 & ASD & $120 / 70$ & 94 & 13 & 1550 & 0 & - & - & 0 & 0 \\
\hline 27 & F 11 & ASD & $29 / 17$ & 22 & 12 & 130 & 0 & - & - & 0 & 0 \\
\hline 28 & F 16 & MS & $63 / 25$ & 39 & 22 & 280 & $+5 / 10$ & - & - & + & + \\
\hline 29 & F 29 & MS & $91 / 42$ & 60 & - & 200 & $-25 / 5$ & - & - & + & + \\
\hline 30 & M 8 & Eisenmenger & $93 / 58$ & 74 & 7 & - & $+10 / 10$ & - & - & + & + \\
\hline 31 & M29 & Pulm. fibrosis & $57 / 36$ & 47 & - & - & $-12 \cdot 5 / 5$ & - & $-20 / 10$ & + & + \\
\hline 32 & M11 & Normal & $35 / 19$ & 26 & 13 & 150 & 0 & - & - & 0 & 0 \\
\hline 33 & F 30 & MS & $28 / 13$ & 18 & 15 & 60 & 0 & - & - & + & + \\
\hline 34 & F 23 & Pulm. hypert. & $85 / 50$ & 63 & - & - & $-20 / 15$ & - & - & 0 & 0 \\
\hline 35 & M 7 & VSD & $26 / 13$ & 20 & 13 & - & & - & - & + & + \\
\hline 36 & F 48 & MS & $52 / 25$ & 38 & 28 & 120 & $-20 / 10$ & - & $\mathbf{0}$ & 0 & 0 \\
\hline 37 & F 24 & MS & $48 / 26$ & 38 & 29 & 110 & 0 & +10 & - & 0 & 0 \\
\hline 38 & F 40 & MS & $160 / 65$ & 100 & 44 & 1210 & 0 & 0 & - & 0 & 0 \\
\hline 39 & F 40 & MS. AI & $38 / 20$ & 27 & 20 & 80 & & 0 & - & $\mathbf{0}$ & $\mathbf{0}$ \\
\hline 40 & F 30 & MS & $108 / 39$ & 77 & & . & $-50 / *$ & - & - & 0 & 0 \\
\hline 41 & F 21 & MS & $38 / 20$ & 29 & 20 & 140 & 0 & - & - & + & + \\
\hline 42 & M39 & Atrial fibr. & $32 / 21$ & 27 & & & 0 & - & - & 0 & 0 \\
\hline 43 & F 40 & MS. AF & $51 / 42$ & 47 & 33 & 290 & 0 & - & - & 0 & 0 \\
\hline 44 & F 53 & ASD & $56 / 21$ & 44 & 14 & 160 & 0 & - & - & $\mathbf{0}$ & $\mathbf{0}$ \\
\hline 45 & M30 & Pulm. hypert. & $113 / 57$ & 80 & 11 & 1780 & 0 & - & - & 0 & 0 \\
\hline 46 & F 34 & MS & $83 / 31$ & 50 & 28 & 260 & $-12 \cdot 5 /^{*}$ & - & - & 0 & 0 \\
\hline 47 & M49 & MS & $73 / 38$ & 50 & 34 & 280 & $-15 /^{*}$ & - & - & 0 & 0 \\
\hline
\end{tabular}

* Right ventricular pressure.

$\dagger$ Patient given atropine 
was taken as indicating the pulmonary arterial systolic pressure. In some patients a double-lumen catheter was used. Acetylcholine was then injected through the side-opening of the catheter and thus arrived in the right ventricle while the pressure in the main pulmonary artery was recorded from the terminal opening.

In nine patients the pulmonary artery wedge pressure was measured via the terminal opening of a doublelumen catheter while acetylcholine was injected into the pulmonary artery via the side opening. In each of these cases, the recording of a true wedge pressure was verified by the shape of the tracing and by the withdrawal of highly oxygenated blood. In nine patients a recording of pressure in a systemic artery was made during the injection of acetylcholine into the right ventricle or pulmonary artery.

The dose of acetylcholine varied from 0.25 to $8.0 \mathrm{mg}$. In those patients in whom an effect was observed, the average effective dose given was $2.3 \mathrm{mg}$. When the effect on the systemic arterial pressure was being observed, the dose of acetylcholine was the maximum that had been used in studying the pressures in the pulmonary circulation in the same patient. There were no recordings of pressure simultaneously in the pulmonary artery or pulmonary wedge position and systemic artery.

Pressures were measured by means of a capacitance manometer, using a direct writing recording apparatus (Bareham). Zero was taken at the level of the table on which the patient lay. In the control observations taken before injection of the drug, the average levels of systolic and diastolic pressures in the pulmonary artery were read during two or more respiratory cycles and the mean pressure was measured by means of planimetry over a similar period of time. The rapid alterations in pressure following the injection of acetylcholine did not allow of so accurate a measurement. In most instances, also, reduced paper speed at this time did not permit measurement of mean pressures. Observations of pressures following the injection of the drug are therefore limited to systolic and diastolic levels and these are given to the nearest $2.5 \mathrm{~mm}$. Hg. Variations in pressure were ignored if they could not be repeated, using the same dose of acetylcholine, after an interval of five minutes.

The evanescent effect of the drug on the pulmonary arterial pressure would have made any simultaneous measurement of pulmonary blood flow impracticable. By using continuous infusions of acetylcholine, it might have been possible to have reached a steady state during which estimations of pulmonary blood flow would have been most valuable. However, the apparatus for carrying this out with sufficient accuracy was not available. In the assessment of resting pulmonary arteriolar resistance made before the injection of acetylcholine, the oxygen uptake was measured from the slope of a spirometric tracing.

\section{RESULTS}

A brief reference to these results has been made previously (Harris, 1955a). Details of each patient are given in Table I.

Pulmonary Arterial Pressures. In twenty-seven patients there was no alteration in the pressure in the pulmonary artery after the injection of acetylcholine. In two there was a slight rise in pressure, but in both this was accompanied by coughing and tachycardia. In eighteen patients, acetylcholine caused a transient fall of varying degree. Fig. 1 shows recordings from a patient in whom the fall was particularly apparent and gives an indication of the reproducibility of the effect. Only in Case 36 was the pressure in the pulmonary artery reduced to within the normal range. The fall in pressure would usually start three or four seconds after the injection and reach its greatest

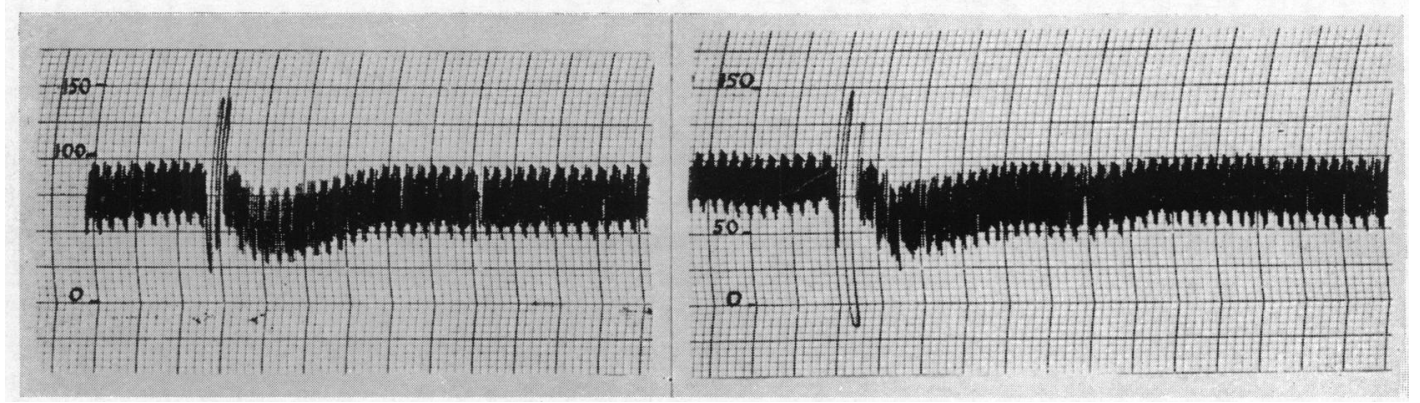

FIG. 1.-Recordings of pulmonary arterial pressure in Case 10. The time of injection of acetylcholine coincides with the sudden wide movements of the recording pen just prior to the fall in pressure. On each occasion, the dose of acetylcholine was $0.25 \mathrm{mg}$. injected into the pulmonary artery. One major horizontal division represents approximately $7 \mathrm{sec}$. The scale of pressures is in $\mathrm{mm}$. $\mathbf{H g}$. 


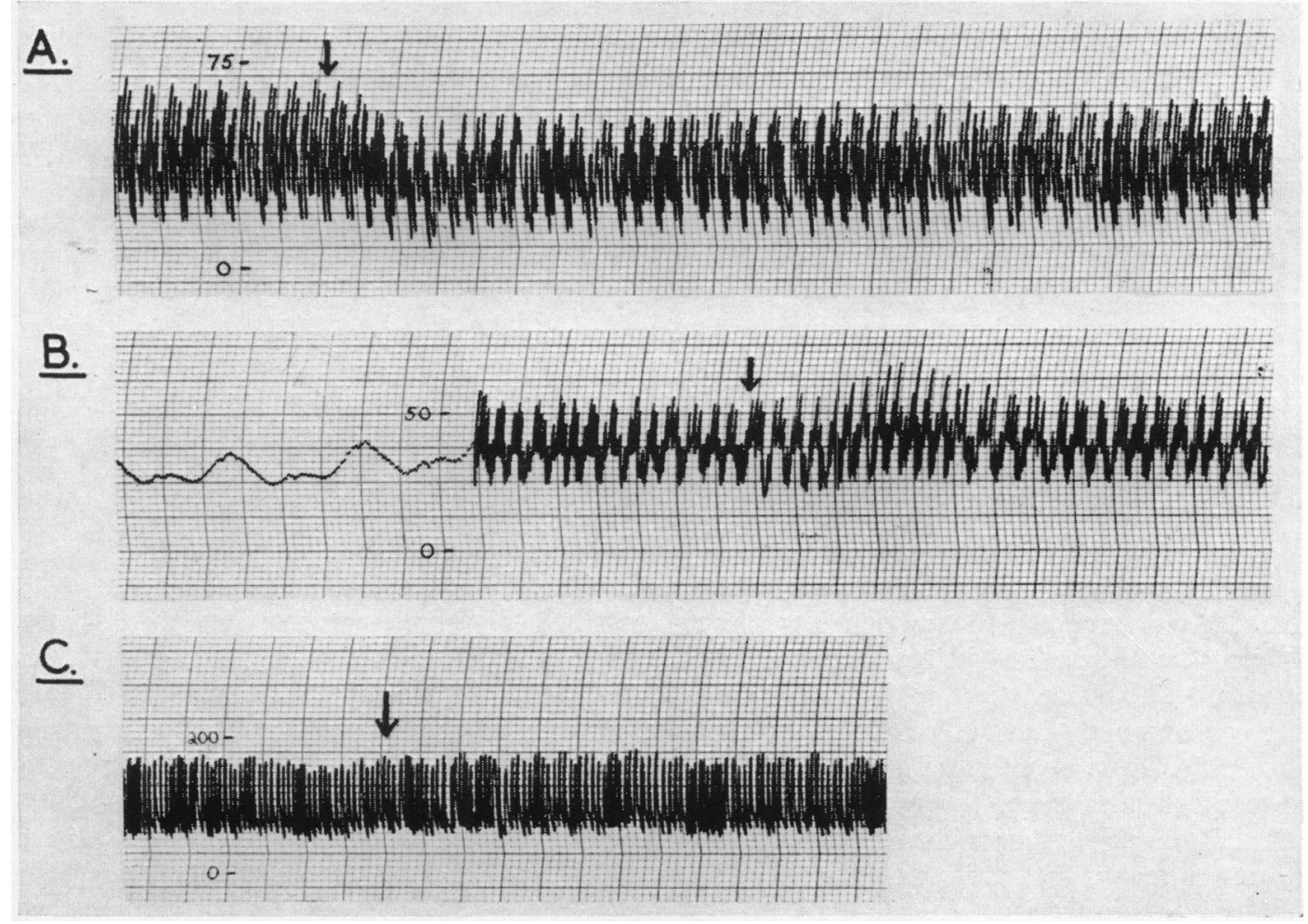

FIG. 2.-The effects of injection of $2 \mathrm{mg}$. acetylcholine in Case 4. (A) Pulmonary arterial pressure. (B) Wedge pressure. (C) Brachial arterial pressure. The arrows indicate the start of the injection. One major horizontal division represents approximately $7 \mathrm{sec}$. The start of tracing (B) is at a fast rate to show the wedge curve; at this point, each major horizontal division represents $0.2 \mathrm{sec}$. The scale of pressure is in $\mathrm{mm}$. $\mathrm{Hg}$.

degree after ten to twenty seconds. The subsequent rise was not so abrupt and might even be prolonged for several minutes (Fig. 2).

Pulmonary Artery Wedge Pressure. In five patients there was no change in the wedge pressure after injection of acetylcholine into the pulmonary artery: in four there was a slight but distinct transient rise (Fig. 2). In the five cases where the wedge pressure was unchanged, the pulmonary arterial pressure was also unaffected by acetylcholine. Of the four cases where the wedge pressure was found to rise, two showed a fall and two showed no change in pulmonary arterial pressure when acetylcholine was injected into the right ventricle. All except one of these nine had mitral stenosis.

Systemic Arterial Pressure. There was no change in the systemic arterial pressure in six, and a transient fall in three patients following the injection of acetylcholine into the right ventricle or pulmonary artery. These nine patients tended to be taken more frequently from those in whom acetylcholine had caused a fall in pressure in the pulmonary artery, since information was being especially sought on how much the fall in pulmonary arterial pressure could be caused by vasodilatation in the systemic circulation. Thus, in seven of them there had been a fall in pulmonary arterial pressure following injection of the drug. Of these seven, only two showed a fall in systemic arterial pressure due to acetylcholine. Of the remaining two in whom the pulmonary arterial pressure had not responded to acetylcholine, the systemic arterial pressure fell in one and was unchanged in the other.

Other Effects. Coughing and tachycardia occurred in many patients when higher doses of acetylcholine were given. These two symptoms occurred together. Often associated with the 
desire to cough was an irritating or burning feeling in the throat or deep inside the chest. On two occasions, the respiratory variation in intravascular pressure in the chest was found to increase following the injection of acetylcholine and this was thought to indicate constriction of the bronchioles. Transient bradycardia such as was described by Carmichael and Fraser (1933) was observed only once. Tachycardia following acetylcholine was, however, common and might persist for several minutes. Abnormality of the electrocardiogram was observed only once when two ventricular premature beats followed the injection, which had been made into the right ventricle. No patient complained of flushing or burning of the skin and, since the investigation was carried out in a red light, it was not possible to observe any reddening of the skin. In no patient did the injection of acetylcholine give any cause for alarm.

In three patients in whom a fall in pulmonary arterial pressure had been caused by the injection of acetylcholine, a similar injection of saline carried out with the same ritual caused no alteration in pressure in the pulmonary artery. The effects of coughing, by itself, on the pulmonary arterial pressure were not comparable with those due to acetylcholine.

\section{Discussion}

From the results quoted above, the pressures in the pulmonary circulation in the majority of cases did not respond to acetylcholine. About one in every three patients, however, showed a transient fall in pulmonary arterial pressure following the injection of the drug, an effect that does not seem to be related to the fall in pressure sometimes found in the systemic arteries. When the wedge pressure has been affected, it has risen. Probably, in these cases, the brief rise in wedge pressure has been made more apparent by the presence of mitral stenosis which was present in all and would delay the speed with which the left ventricle could restore the left atrial pressure.

A fall in pulmonary arterial pressure accompanied by a rise in wedge pressure cannot be accounted for by a reduction in pulmonary blood volume due to systemic vasodilatation. Neither would bronchoconstriction be expected to have this effect on the pressures in the pulmonary circulation. A reduction in cardiac output is unlikely to have given rise to so immediate a fall in pulmonary arterial pressure, but in the absence of measurement this must be accepted as possible. The fall in pulmonary arterial pressure has not been due to a bradycardia such as was observed in dogs by Johnson et al. (1937).

There would seem, therefore, to be strong indications that acetylcholine has caused a vasodilatation in the branches of the pulmonary artery. Why, however, should such vasodilatation occur in some patients and not in others? A clue to the answer may be given by the histogram in Fig. 3 where the patients are arranged according to the mean pulmonary arterial pressure that had been measured before giving the drug. When the pressure in the pulmonary artery was normal, a fall in pressure following the injection was never found. As the mean pressure in the pulmonary artery rose, there was a gradual increase in the proportion of patients showing a fall in pressure until, between mean pressures of 50-70 $\mathrm{mm}$. $\mathrm{Hg}$, almost every case showed a response. Thereafter, the proportion of patients showing a response fell off and all the patients within the higher ranges of pulmonary arterial pressure were once more unresponsive.

This peculiar distribution of the responsive group within the total series of patients has been tested by means of the $\chi^{2}$ test, and would arise by chance less often than once in a hundred times. Further analyses, the details of which are given elsewhere (Harris, 1955b), showed that the type of disease or the age or sex of the patient had no specific influence on the action of acetylcholine. It seems, therefore, that the responsiveness of the pulmonary circulation to the action of acetylcholine is related in a distinctive way to the initial level of pressure in the pulmonary artery.

The wide differences in pulmonary arterial pressure that occur between the various patients are mainly due to differences in the resistance of flow offered by the smaller branches of the pulmonary artery, so that the effects of acetylcholine are most probably related primarily to the nature of the resistance occurring at this level. The development of pulmonary arterial hypertension is accompanied by distinctive changes in the structure of the small pulmonary arteries. Thus, the 


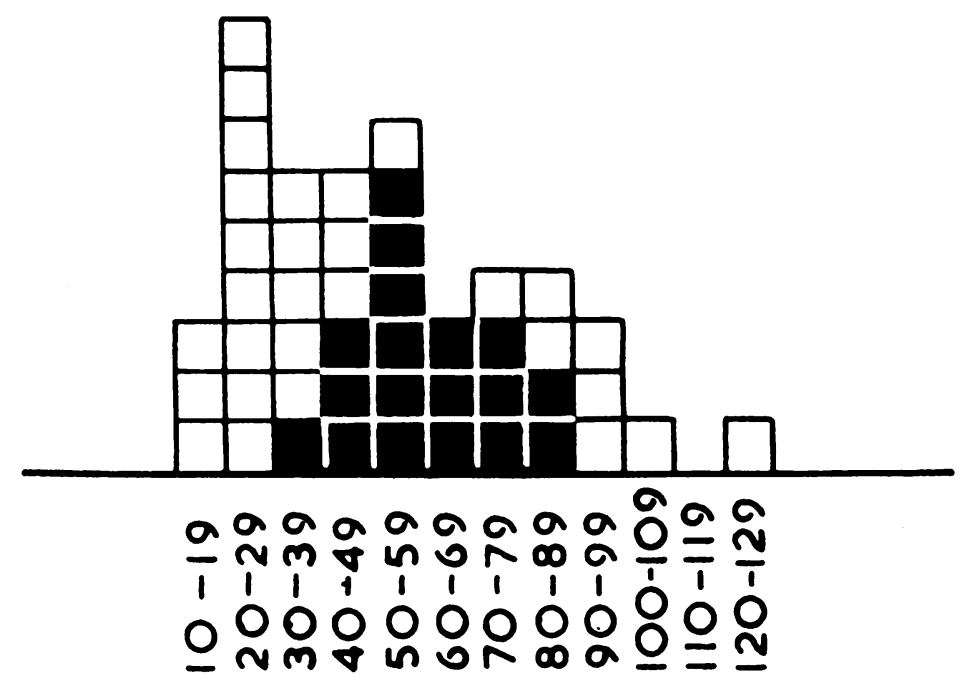

PULM. ART. PRESS. MM. HG.

FIG. 3.- Histogram, showing the patients arranged according to the level of the initial mean pressure in the pulmonary artery. Each black square indicates a patient in whom the pressure in the pulmonary artery fell after acetylcholine had been given. Each white square represents a patient in whom it did not show such a fall.

responsiveness or unresponsiveness of these vessels and the degree of resistance that they offer to flow may well both be linked to the anatomical differences to be found in their walls.

Unfortunately, no such direct comparison between function and structure can be made in these patients, since the microscopical structure of the small pulmonary arteries is known in only three of them. On the other hand, the special relationship of the response to acetylcholine to the level of pulmonary arterial pressure seems not to be a matter of chance, and it may therefore be allowable to compare this functional relationship with the variations in structure that are known to occur in the small pulmonary arteries during disease.

In this comparison, the alterations in structure of the media of the small pulmonary arteries would be expected to be of prime importance, since any sudden variations in the calibre of these vessels are most likely to be due to a change in the tone of the muscle contained in their walls. Normally, the small pulmonary arteries are only meagerly provided with muscle (Brenner, 1935), but, in those diseases where pulmonary hypertension occurs, a thickening of the medial muscle of these vessels has been frequently observed (Parker and Weiss, 1936; Civin and Edwards, 1950; Selzer and Laqueur, 1951; Henry, 1952; Brown et al., 1955). It is likely that such hypertrophy is in itself evidence of an active vasoconstriction occurring intermittently or continuously for a prolonged time (Harris, 1955a). Eventually, however, fibrosis develops in the walls of the small pulmonary arteries, tending to replace the muscular tissue (Kaump and Dry, 1938; Larrabee et al., 1949).

It may be possible, therefore, to explain the relationship of the effects of acetylcholine on the pulmonary circulation to the level of the pulmonary arterial pressure in terms of these two overlapping processes of muscular hypertrophy and fibrosis which take place in the walls of the small pulmonary arteries. When the pulmonary arterial pressure is normal, the muscle of the media of these vessels is thin and its tone slight, so that the response to acetylcholine is too feeble to have any substantial effect on the pressure in the pulmonary artery. When the pulmonary arterial pressure becomes raised, the medial muscle becomes hypertrophied and its tone is probably increased: the activity of acetylcholine will then become increasingly apparent since the muscular coat is capable of more effective contraction and relaxation. Finally, with predominant fibrosis the small pulmonary arteries gradually become rigid tubes, once more unable to respond to the drug.

Other factors might influence these effects of acetylcholine at different levels of pressure. It is likely, for instance, that in normal people the effects of vaso-active drugs on the pressure in the pulmonary artery 
are reduced by the opening or closing of alternative channels for the passage of blood through the lungs. This cushioning effect may gradually be lost in the presence of an increasing pulmonary hypertension since it is possible that the level of pressure in the pulmonary artery then comes to exceed by an increasing amount the critical opening and closing pressures of the small arterial branches. Further, although the same fractional change in resistance should produce an equal fractional change in pulmonary arterial pressure, yet the absolute change in pulmonary arterial pressure will naturally increase as the initial level of pressure becomes higher. Further, it might be argued that subintimal thickening, by encroaching upon the size of the lumen of a small pulmonary artery, would actually magnify any fractional alterations in the size of the lumen following activity of the media. All of these factors could play a part in increasing the effects of acetylcholine in pulmonary hypertension, though none can explain the final diminution of the effect of acetylcholine when the pulmonary arterial pressure becomes extremely high.

The exact way in which acetylcholine might cause pulmonary vasodilatation is not revealed by these observations. Its rapid action on the pulmonary arterial pressure suggests that vasodilatation in the pulmonary circuit is most probably due to a direct influence of the drug on the muscle fibres in the walls of the vessels. One of the three patients who had been given atropine had a substantial fall in pulmonary arterial pressure after acetylcholine, and it seems likely, therefore, that the doses of acetylcholine used were but little inhibited by a therapeutic concentration of atropine in the tissues.

\section{SUMMARY}

The rapid injection of acetylcholine into the pulmonary artery or right ventricle has caused a transient fall of the pulmonary arterial pressure in 18 of 47 patients with various abnormalities. There is strong evidence that this fall was due to vasodilatation in the periphery of the pulmonary arterial tree. The response to acetylcholine was related to the initial level of pulmonary arterial pressure in a distinctive manner. It was found most frequently in patients with a moderate rise of pulmonary arterial pressure and did not occur when this was normal or when it was greatly raised. These results may be related to the structural alterations that occur in the small pulmonary arteries in association with pulmonary hypertension.

I am most grateful to Dr. Terence East for advice and encouragement.

\section{REFERENCES}

Bareham, F. R. To be published.

Brown, J. W., Heath, D., and Whitaker, W. (1955). Brit. Heart J., 17, 273.

Carmichael, E. A., and Fraser, F. R. (1933). Heart, 16, 262.

Civin, W. H., and Edwards, J. E. (1950). Circulation (N.Y.), 2, 545.

Dale, H. H. (1914). J. Pharmacol., 6, 147.

Daly, I. de B. (1933). Physiol. Rev., 13, 149.

and von Euler, U. S. (1932). Proc. Roy. Soc. B., 110, 92.

Dirken, M. N. J., and Heemstra, H. (1948). Quart J. exper. Physiol., 34, 227.

Ellis, L. B., and Weiss, S. (1932). J. Pharmacol., 44, 235.

von Euler, U. S. (1932). J. Physiol., 74, 271.

and Liljestrand, G. (1946). Acta. physiol. Scand., 12, 301.

Foggie, P. (1940). Quart. J. exp. Physiol., 26, 225.

Franklin, K. J. (1932). J. Physiol., 75, 471.

Friedberg, L., Katz, L. N., and Steinitz, F. S. (1943). J. Pharmacol. exper. Therap., 77, 80.

Gaddum, J. H., and Holtz, P. (1933). J. Physiol., 77, 139.

Hamilton, W. F., Woodbury, R. A., and Vogt, E. (1939). Amer. J. Physiol., 125, 130.

Harris, P. (1955a). Brit. Heart J., 17, 85. $(1955 b)$. University of London Thesis.

Hunt, R. (1917). Amer. J. Physiol., 45, 197 and 231.

, and Taveau, R. de M. (1906). Brit. med. J., 2, 1788.

Johnson, V., Hamilton, W. F., Katz, L. N., and Weinstein, W. (1937). Amer. J. Physiol., $120,624$.

Kaump, D. H., and Dry, T. J. (1938). Arch. intern. Med., 61, 1.

Larrabee, W. F., Parker, R. L., and Edwards, J. E. (1949). Proc. Mayo Clin., 24, 316.

Petrovskaia, B. (1939). Quart. J. exper. Physiol., 29, 277.

Selzer, A., and Laqueur, G. L. (1951). Arch. intern. Med., 87, 218.

Tiffenau, R., and Beauvallet, M. (1944). Compts. rendu. Soc. Biol., Paris, 138, 747. 